\title{
ON STABILITY AND STATIONARY POINTS IN NONLINEAR OPTIMIZATION
}

\author{
J. GUDDAT ${ }^{1}$, H. TH. JONGEN ${ }^{2}$ AND J. RUECKMANN ${ }^{3}$
}

(Received 26 June 1985; revised 8 November 1985)

\begin{abstract}
This paper presents three theorems concerning stability and stationary points of the constrained minimization problem:

$$
\operatorname{Min} f(x) \text { on } M[H, G]=\left\{x \in \mathbf{R}^{n} \mid H(x)=0, G(x) \geqslant 0\right\} .
$$

In summary, we prove $(\mathscr{A})$ that, given the Mangasarian-Fromovitz constraint qualification (MFCQ), the feasible set $M[H, G]$ is a topological manifold with boundary, with specified dimension; ( $\mathscr{B})$ a compact feasible set $M[H, G]$ is stable (perturbations of $H$ and $G$ produce homeomorphic feasible sets) if and only if MFCQ holds; $(\mathscr{C})$ under a stability condition, two lower level sets of $f$ with a Kuhn-Tucker point between them are homotopically related by attachment of a $k$-cell ( $k$ being the stationary index in the sense of Kojima).
\end{abstract}

\section{Introduction, main results}

Let $\mathbf{R}^{n}$ be the $n$-dimensional Euclidean space and $C^{k}\left(\mathbf{R}^{n}, \mathbf{R}\right)$ the space of real valued, $k$-times continuously differentiable functions on $\mathbf{R}^{n}$. For $r \leqslant k$ the space $C^{k}\left(\mathbf{R}^{n}, \mathbf{R}\right)$ will be topologized by means of the strong $C^{r}$-topology (or $C^{r}$-Whitney topology), denoted by $C_{S}^{r}$ (cf. [8]).

In fact, the $C_{S}^{r}$ topology is generated by allowing perturbations of the functions and their derivatives up to order $r$ which are controlled by continuous positive functions $\varepsilon():. \mathbf{R}^{n} \rightarrow \mathbf{R}$ (rather than only constants $\varepsilon$ ). In particular, the $C_{S}^{r}$ topology takes asymptotical effects (at infinity) into account since the infimum of

\footnotetext{
${ }^{1}$ Humboldt-University, Berlin, German Democratic Republic

${ }^{2}$ Twente University of Technology, Enschede, The Netherlands

${ }^{3}$ Technical University Leipzig, Leipzig, German Democratic Republic

(C) Copyright Australian Mathematical Society 1986, Serial-fee code 0334-2700/86
} 
$\varepsilon(x)$ might be zero. Let us fix the dimension $n$ and two finite index sets $I, J$, with $I=\{1, \ldots, m\}, J=\{1, \ldots, s\}$ and $m<n$.

Unless stated otherwise, we will assume throughout that the functions $f, h_{i}, g_{j}$, $i \in I, j \in J$ belong to $C^{1}\left(\mathbf{R}^{n}, \mathbf{R}\right)$. By means of capital $H$, resp. $G$, we denote $\left(h_{1}, \ldots, h_{m}\right)$, resp. $\left(g_{1}, \ldots, g_{s}\right)$.

The optimization problems under consideration will be of the following standard type:

$$
\mathscr{P}: \text { Minimize } f \text { on } M[H, G],
$$

where the feasible set $M[H, G]$ is defined as

$$
M[H, G]=\left\{x \in \mathbf{R}^{n} \mid h_{i}(x)=0, g_{j}(x) \geqslant 0, i \in I, j \in J\right\} .
$$

In case we wish to ignore the equality constraints $(H)$, resp. the inequality constraints $(G)$, we simply write $M[G]$, resp. $M[H]$, instead of $M[H, G]$. For $x \in \mathbf{R}^{n}$, we denote the set of active (= binding) inequality constraints by $J_{0}(\bar{x})$, i.e.:

$$
J_{0}(x)=\left\{j \in J \mid g_{j}(x)=0\right\} .
$$

Let $\bar{x} \in M[H, G]$ be a local minimum point for $\left.f\right|_{M[H, G]}$ If, additionally, an appropriate constraint qualification holds at $\bar{x}$, we have a relation of the following type (cf. [4]):

$$
\left.\begin{array}{r}
D f=\sum_{i \in I} \lambda_{i} D h_{i}+\left.\sum_{\substack{j \in J_{0}(\bar{x}) \\
\mu_{j}}} \mu_{j} D g_{J}\right|_{x=\bar{x}}, j \in J_{0}(\bar{x})
\end{array}\right\} .
$$

If relation (1.4) holds at $\bar{x}$, then we call $\bar{x}$ a Kuhn-Tucker point.

In (1.4), $D f$ stands for the row-vector of first partial derivatives. A condition on the set $\left\{D f, D h_{\imath}, D g_{j}, i \in I, j \in J_{0}(\bar{x})\right\}$ at $\bar{x}$ which is both necessary and sufficient in order that the local minimum $\bar{x}$ is a Kuhn-Tucker point is given in [7, Theorem 4.2].

Two constraint qualifications play an essential role in this study, namely, the linear independence condition (LI) and the Mangasarian-Fromovitz constraint qualification (MFCQ) at an $\bar{x} \in M[H, G]$ :

(LI) The linear independence condition is said to hold at $\bar{x}$ if the vectors $D h_{i}(\bar{x}), D g_{,}(\bar{x}), i \in I, j \in J_{0}(\bar{x})$ are linearly independent.

(MFCQ) The Mangasarian-Fromovitz constraint qualification is said to hold at $\bar{x}$ if the following two conditions are satisfied:

MF 1 . The vectors $D h_{i}(\bar{x}), i \in I$, are linearly independent. 
MF 2. There exists a vector $\xi \in \mathbf{R}^{n}$ satisfying:

$$
\begin{cases}D h_{i}(\bar{x}) \xi=0, & i \in I, \\ D g_{j}(\bar{x}) \xi>0, & j \in J_{0}(\bar{x}) .\end{cases}
$$

A vector $\xi$ satisfying 1.5 .a, b will be called an $M F$-vector at $\bar{x}$.

Obviously, (LI) implies (MFCQ). If (LI) is satisfied at all points of the feasible set, then $M[H, G]$ is a $\left(C^{1}-\right)$ manifold with corners, or a regular constraint set in the terminology of [11]. In particular, $M[H, G]$ is locally diffeomorphic to $\mathbf{R}^{p} \times \mathbf{H}^{q}, \mathbf{H}^{q}$ being the nonnegative orthant of $\mathbf{R}^{q}$, where $p=n-|I|-\left|J_{0}(\bar{x})\right|$ and $q=\left|J_{0}(\bar{x})\right|$. Consequently, $M[H, G]$ is a topological manifold with boundary (i.e., locally homeomorphic to $\left.\mathbf{R}^{k} \times \mathbf{H}^{1}, k=n-|I|-1\right)$. In the case that (MFCQ) is satisfied at all points of $M[H, G]$, but not necessarily (LI), the situation becomes much more complicated.

However, as a first result we state:

THEOREM A (Manifold Theorem). Suppose that (MFCQ) is satisfied at all points $x \in M[H, G]$. Then $M[H, G]$ is a topological manifold with boundary $\partial M[H, G]=$ $\left\{x \in \mathbf{R}^{n} \mid h_{i}(x)=0, i \in I, \min _{j \in J} g_{j}(x)=0\right\}$ and the dimension of $M[H, G]$ equals $n-|I|$.

The Mangasarian-Fromovitz constraint qualification plays an important role in sensitivity analysis; see e.g. the survey-paper of Fiacco and Kyparisis [3]. In particular, if $\bar{x}$ is a Kuhn-Tucker point for $\left.f\right|_{M[H, G]}$, then the set of admissible Lagrange parameters $\left(\lambda_{i}, i \in I, \mu, j \in J_{0}(\bar{x})\right)$ satisfying (1.4) is bounded if and only if (MFCQ) holds at $\bar{x}$ (Gauvin's result [5]). Further, Kojima was able to characterize strong stability of Kuhn-Tucker points under the (MFCQ) with the aid of the derivatives of the appearing functions up to second order (cf. [17]). We will return to this point later. Next, Jongen, Jonker and Twilt considered generic one-parameter families of sets defined by (in)equality constraints ([10]). They classified the possible singularities and studied their topological consequences. As a special result it turned out-under compactness assumptions-that the failure of (MFCQ) is necessary for a bifurcation of the sets under consideration.

Kojima and Hirabayashi studied optimization problems depending on one parameter, again under (MFCQ), and it turned out that, in general, the KuhnTucker set (one-dimensional) is pieced together from differentiable curves ([18]). The failure of (MFCQ) may give rise to boundary points of the Kuhn-Tucker manifold (cf. [12], [13], [23]). Another interesting result where the (MFCQ) plays an essential role is the following.

In E. S. Levitin [19] and B. Bank et al. [1] a parameter-dependent feasible set

$$
M(\lambda):=\left\{x \in \mathbf{R}^{n} \mid h_{i}(x, \lambda)=0, i \in I, g_{j}(x, \lambda) \geqslant 0, j \in J, \lambda \in \mathbf{R}^{k}\right\}
$$


is considered where $h_{i}, g_{j} \in C^{1}\left(\mathbf{R}^{n} \times \mathbf{R}^{k}, \mathbf{R}\right), i \in I, j \in J$. Let $\lambda^{0} \in \mathbf{R}^{k}$ where $M\left(\lambda^{0}\right) \neq \varnothing, x^{0} \in M\left(\lambda^{0}\right)$ and

$$
\begin{aligned}
M^{\mathscr{L}}(\lambda):=\left\{\xi \in \mathbf{R}^{n} \mid D_{x} h_{i}\left(x^{0}, \lambda^{0}\right) \xi=-D_{\lambda} h_{i}\left(x^{0}, \lambda^{0}\right)\left(\lambda-\lambda^{0}\right), i \in I,\right. \\
\\
\left.D_{x} g_{j}\left(x^{0}, \lambda^{0}\right) \xi \geqslant-D_{\lambda} g_{j}\left(x^{0}, \lambda^{0}\right)\left(\lambda-\lambda^{0}\right), j \in J_{0}\left(x^{0}, \lambda^{0}\right)\right\} .
\end{aligned}
$$

Then we have the following statement under (MFCQ) at $\left(x^{0}, \lambda^{0}\right)$ (E. S. Levitin [19], Theorem 6.3.3 in B. Bank et al. [1]):

Each selection function $y$ of the point-to-set mapping $M^{\mathscr{L}}$ that is locally Lipschitzian at $\lambda^{0}$ and satisfies $y\left(\lambda^{0}\right)=0$ fulfils

$$
d\left(x^{0}+y(\lambda), M(\lambda)\right)=o\left(\left\|\lambda-\lambda^{0}\right\|\right),
$$

where $d$ denotes the distance (cf. the subsequent formula (1.6)).

Finally, in [22] S. M. Robinson already studied local stability of the feasible set in relation with (MFCQ). All these results gave us the feeling that (MFCQ) is very closely related to the global stability of the feasible set. This will become clear in Theorem B, but first we need a definition. The $C_{S}^{r}$-topology for a number of copies of $C^{k}\left(\mathbf{R}^{n}, \mathbf{R}\right)$ will be the product-topology; in particular, $C^{k}\left(\mathbf{R}^{n}, \mathbf{R}\right)^{m}=$ $C^{k}\left(\mathbf{R}^{n}, \mathbf{R}\right) \times \cdots \times C^{k}\left(\mathbf{R}^{n}, \mathbf{R}\right)(m$-times $)$.

Definition 1.1. Let $h_{i} \in C^{2}\left(\mathbf{R}^{n}, \mathbf{R}\right), i \in I$. The set $M[H, G]$ is called stable if there exists a $C_{S}^{1}$-neighborhood $\mathcal{O}$ of $(H, G)$ in $C^{2}\left(\mathbf{R}^{n}, \mathbf{R}\right)^{m} \times C^{1}\left(\mathbf{R}^{n}, \mathbf{R}\right)^{s}$ such that for every $(\tilde{H}, \tilde{G}) \in \mathcal{O}$ the corresponding set $M[\tilde{H}, \tilde{G}]$ is homeomorphic with $M[H, G]$.

Theorem B (Stability Theorem). Let $h_{i} \in C^{2}\left(\mathbf{R}^{n}, \mathbf{R}\right), i \in I$, and suppose that $M[H, G]$ is compact. Then $M[H, G]$ is stable if and only if (MFCQ) holds at every point $x \in M[H, G]$.

Now we return to the concept of strongly stable Kuhn-Tucker points (= strongly stable stationary solutions) as introduced by Kojima. We refer to [17] for definitions etc.; in [2] a different, but equivalent characterization of this concept is given under (LI). To a strongly stable Kuhn-Tucker point, Kojima associated a nonnegative integer called the stationary index (s. index); moreover, he mentioned in his basic paper [17] that the s. index is closely related to the Morse-index [20]. We will show that, indeed, the s. index is precisely the Morse index in the sense of the subsequent deformation theorem (Theorem C). Our result is directly connected with the topology of lower level sets, which, in our opinion is more adequate with respect to optimization than the rougher information obtained by exploiting the degree of an associated mapping (cf. [17]). 
It is important to note the following basic fact (cf. [17]):

The existence and local uniqueness of a strongly stable Kuhn-Tucker point as well as its s. index are preserved under local $C^{2}$-perturbations of the underlying functions $f, h_{i}, g_{j}$.

In order to state Theorem $\mathrm{C}$ we need some further definitions.

Let $A \subset \mathbf{R}^{n}$ be nonempty and $\bar{x} \in \mathbf{R}^{n}$. By $d(\bar{x}, A)$ we denote the distance from $\bar{x}$ to $A$, i.e.,

$$
d(\bar{x}, A)=\inf _{x \in A}\|x-\bar{x}\| .
$$

For our purpose, we define the normal cone $N_{\bar{x}}$ at $\bar{x} \in M[H, G]$ as follows:

$$
\begin{aligned}
& N_{\bar{x}}=\left\{\sum_{i \in I} \lambda_{i} D h_{i}(\bar{x})\right. \\
&\left.+\sum_{j \in J_{0}(\bar{x})} \mu_{j} D g_{j}(\bar{x}) \mid \lambda_{i}, \mu_{J} \in \mathbf{R}, \mu_{J} \geqslant 0, i \in I, j \in J_{0}(\bar{x})\right\} \cup\{0\} .
\end{aligned}
$$

The next condition is the appropriate generalization of the Palais-Smale "Condition C" ([21]) as a substitute of the possible lack of compactness of the feasible set (cf. also [11]).

Condition $C^{*}$. The function $f$ is said to fulfil Condition $\mathrm{C}^{*}$ on $M[H, G]$ with respect to the interval $[a, b]$ if, for any closed subset $S$ of $f^{-1}([a, b]) \cap M[H, G]$ that does not contain Kuhn-Tucker points for $\left.f\right|_{M[H, G]}$, we have

$$
\inf \left\{d\left(D f(x), N_{x}\right) \mid x \in S\right\}>0 .
$$

For a subset $M \subset \mathbf{R}^{n}, a, b \in \mathbf{R}$ and a given function $f$ we define:

$$
M^{a}=\{x \in M \mid f(x) \leqslant a\}, M_{a}^{b}=\{x \in M \mid a \leqslant f(x) \leqslant b\} .
$$

In the next theorem we use several topological concepts; see [11], [24] for a detailed exposé.

Theorem C (Deformation Theorem). Let $a, b \in \mathbf{R}$ and $a<b$. Suppose that (MFCQ) holds at all points $x \in M_{a}^{b}$, with $M:=M[H, G]$, and that $f$ fulfils Condition $\mathrm{C}^{*}$ on $M$ with respect to $[a, b]$.

a. If $h_{i} \in C^{2}\left(\mathbf{R}^{n}, \mathbf{R}\right), i \in I$, and $M_{a}^{b}$ contains no Kuhn-Tucker point, then $M^{a}$ is a strong deformation retract of $M^{b}$.

b. Let $f, h_{i}, g_{j} \in C^{2}\left(\mathbf{R}^{n}, \mathbf{R}\right), i \in I, j \in J$. Suppose, in addition, that $M_{a}^{b}$ contains exactly one Kuhn-Tucker point $\bar{x}$ with $a<f(\bar{x})<b$ and suppose that $\bar{x}$ is strongly stable with $s$. index equal to $k$. Then, $M^{b}$ is homotopy-equivalent to $M^{a}$ with a $k$-cell attached. 
The present paper is organized as follows. Section 2 contains a list of lemmas and preliminary results. In Section 3 we prove the above theorems and give additional comments. Finally, in Section 4 we present some consequences.

\section{Lemmas and preliminary results}

LEMMA 2.1. The Mangasarian-Fromovitz constraint qualification is invariant under local $C^{1}$-coordinate transformations.

Proof. Let $h_{l}, g_{j} \in C^{1}\left(\mathbf{R}^{n}, \mathbf{R}\right), i \in I, j \in J$ and suppose that $\xi$ is an $M F$-vector at $\bar{x} \in M[H, G]$. Moreover, let $\mathcal{O}, \mathscr{V} \subset \mathbf{R}^{n}$ be open, $\bar{x} \in \mathcal{O}$ and $\Phi: \mathcal{O} \rightarrow \mathscr{V}$ be a $C^{1}$-diffeomorphism sending $\bar{x}$ to $\bar{y}$. Define $\tilde{h}_{i}(y)=h_{l} \circ \Phi^{-1}(y), i \in I$ and $\tilde{g}_{j}$ similarly. Then, $\tilde{h}_{i}, \tilde{g}_{j}$ are of class $C^{1}$ on $\mathscr{V}$ and since $D \Phi^{-1}(\tilde{y}) \cdot D \Phi(\bar{x})=$ Identity, we see that the vector $\tilde{\xi}:=D \Phi(\bar{x}) \xi$ is an $M F$-vector at $\bar{y}$ with $H, G$ replaced by $\tilde{H}, \tilde{G}$.

Remark 2.1. Let $\bar{x} \in M[H, G]$ and suppose that (MFCQ) holds at $\bar{x}$. Then, for a local analysis, we may delete the equality constraints since the common zero set of $h_{i}, i \in I$, is a $C^{\mathbf{l}}$-manifold in a neighborhood of $\bar{x}$. In fact, choose $\xi, \in \mathbf{R}^{n}$, $j=m+1, \ldots, n$, such that the vectors $D^{T} h_{i}(\bar{x}), i \in I=\{1, \ldots, m\}, \xi_{j}, j=$ $m+1, \ldots, n$ form a basis for $\mathbf{R}^{n}$. Put

$$
y=\Phi(x), \text { where }\left\{\begin{array}{l}
y_{t}=h_{i}(x), \quad i=1, \ldots, m \\
y_{j}=\xi_{j}^{T}(x-\bar{x}), \quad j=m+1, \ldots, n .
\end{array}\right.
$$

Then $\Phi$ is of class $C^{1}$ and the Jacobian matrix $D \Phi(\bar{x})$ is nonsingular. Consequently, $\Phi$ is locally invertible and hence a local $C^{1}$-coordinate transformation sending $\bar{x}$ to the origin. Now the common zero set of the functions $h_{i}, i \in I$ is locally transformed under $\Phi$ to the set $\{0\} \times \mathbf{R}^{n-m}, 0 \in \mathbf{R}^{m}$. Moreover, if all $h_{i}$ are of class $C^{k}, k \geqslant 1$, then $\Phi$ is also of class $C^{k}$. Lemma 2.1. implies that (MFCQ) remains valid in the new coordinates.

The proof of the next lemma only depends on a continuity argument and will be omitted.

LEMMA 2.2. Suppose that (MFCQ) is satisfied at all points $x \in M[H, G]$. Then, for every neighborhood $\mathcal{O}$ of the (closed) set $M[H, G]$ there exists a $C_{S}^{1}$-neighborhood $\mathscr{V}$ of $(H, G)$ in $C^{1}\left(\mathbf{R}^{n}, \mathbf{R}\right)^{m+s}$ such that every $(\tilde{H}, \tilde{G}) \in \mathscr{V}$ satisfies:

(i) $M[\tilde{H}, \tilde{G}] \subset \mathcal{O}$,

(ii) (MFCQ) holds at every point $x \in M[\tilde{H}, \tilde{G}]$. 
Lemma 2.3. Let $f \in C^{1}\left(\mathbf{R}^{n}, \mathbf{R}\right)$ and $\bar{x} \in \mathbf{R}^{n}$. Then, every $C_{S}^{1}$-neighborhood of $f$ contains an $\tilde{f}$ satisfying:

(i) $\tilde{f} \in C^{\infty}\left(\mathbf{R}^{n}, \mathbf{R}\right)$,

(ii) $\tilde{f}(\bar{x})=f(\bar{x}), D \tilde{f}(\bar{x})=D f(\bar{x})$.

The proof of Lemma 2.3(i) follows from the fact that $C^{\infty}\left(\mathbf{R}^{n}, \mathbf{R}\right)$ is $C_{S}^{1}$-dense in $C^{1}\left(\mathbf{R}^{n}, \mathbf{R}\right)$ (cf. [8]); for the validity of (ii) see e.g. [14], [16].

The proof of the following two lemmas (Lemma 2.4, 2.5) is based on smooth approximation (cf. Lemma 2.3) and subsequently local perturbations with constants resp. linear functions, thereby exploiting Sard's Theorem. Details can be found in [15], [16] and they will be omitted here.

Let $h_{i}, g_{j} \in C^{1}\left(\mathbf{R}^{n}, \mathbf{R}\right), i \in I, j \in J$ and $C_{1}, C_{2} \subset \mathbf{R}^{n}$ be disjoint closed subsets. Let $(\tilde{H}, \tilde{G}) \in C^{1}\left(\mathbf{R}^{n}, \mathbf{R}\right)^{m+s}$ belong to $\mathscr{F}_{1}\left(C_{1}, C_{2}\right)$ if and only if

(i) $\tilde{H}, \tilde{G}$ coincide with $H, G$ on $C_{1}$,

(ii) $M[\tilde{H}, \tilde{G}]$ satisfies (LI) at all $x \in M[\tilde{H}, \tilde{G}] \cap C_{2}$.

LEMMA 2.4. $\mathscr{F}_{1}\left(C_{1}, C_{2}\right)$ intersects every $C_{S}^{1}$-neighborhood of $(H, G)$ and $\mathscr{F}_{1}\left(\varnothing, C_{2}\right)$ is $C_{S}^{1}$-open.

Definition 2.1. (cf. [11]). Let $f, h_{\imath}, g_{j} \in C^{2}\left(\mathbf{R}^{n}, \mathbf{R}\right), i \in I, j \in J$. Let $\bar{x} \in$ $M[H, G]$ and suppose that (LI) holds at $\bar{x}$. Then $\bar{x}$ is called a critical point for $\left.f\right|_{M[H, G]}$ if the following relation (with unique $\bar{\lambda}_{i}, \bar{\mu}_{j}$ ) holds:

$$
D f=\sum_{i \in I} \bar{\lambda}_{i} D h_{i}+\left.\sum_{j \in J_{0}(\bar{x})} \bar{\mu}_{j} D g_{j}\right|_{x=\bar{x}} .
$$

Moreover, $\bar{x}$ is a nondegenerate critical point if ND1, ND2 are satisfied $\left(\bar{\lambda}_{i}, \bar{\mu}_{j}\right.$ as in (2.2)):

(ND2)

$$
\bar{\mu}_{j} \neq 0, j \in J_{0}(\bar{x}),
$$

$V^{T} D^{2} L(\bar{x}) V$ is nonsingular,

where $L(x)=f(x)-\sum_{i \in I} \bar{\lambda}_{i} h_{i}(x)-\sum_{j \in J_{0}(\bar{x})} \bar{\mu}_{j} g_{j}(x)$ (Lagrange-function), $D^{2} L$ denotes the matrix of partial derivatives of second order, and where $V$ is a matrix of $n$-vectors whose columns form a basis for the tangent space

$$
T:=\left\{\xi \in \mathbf{R}^{n} \mid D h_{i}(\bar{x}) \xi=0, D g_{j}(\bar{x}) \xi=0, i \in I, j \in J_{0}(\bar{x})\right\} .
$$

The number of negative $\mu_{j}$ resp. negative eigenvalues of $V^{T} D^{2} L(\bar{x}) V$ is called the linear resp. quadratic index of the nondegenerate critical point $\bar{x}$.

Let $f, h_{i}, g_{j} \in C^{2}\left(\mathbf{R}^{n}, \mathbf{R}\right), i \in I, j \in J$ and $C_{1}, C_{2}$ be disjoint closed subsets of $\mathbf{R}^{n}$. Let $(\tilde{f}, \tilde{H}, \tilde{G}) \in C^{2}\left(\mathbf{R}^{n}, \mathbf{R}\right)^{1+m+s}$ belong to $\mathscr{F}_{2}\left(C_{1}, C_{2}\right)$ if and only if

(i) $\tilde{f}, \tilde{H}, \tilde{G}$ coincide with $f, H, G$ on $C_{1}$,

(ii) $M[\tilde{H}, \tilde{G}]$ satisfies (LI) at all points $x \in M[\tilde{H}, \tilde{G}] \cap C_{2}$,

(iii) on $M[\tilde{H}, \tilde{G}] \cap C_{2}$ all critical points for $\left.\tilde{f}\right|_{M[\tilde{H}, \tilde{G}]}$ are nondegenerate. 
LEMMA 2.5. $\mathscr{F}_{2}\left(C_{1}, C_{2}\right)$ intersects every $C_{S}^{2}$-neighborhood of $(f, H, G)$ and $\mathscr{F}_{2}\left(\varnothing, C_{2}\right)$ is $C_{S}^{2}$-open.

The next lemma is almost obvious.

Lemma 2.6. Let $f, h_{i}, g_{j} \in C^{2}\left(\mathbf{R}^{n}, \mathbf{R}\right), i \in I, j \in J$. Let $\bar{x} \in M[H, G]$ and suppose that (LI) holds at $\bar{x}$. If $\bar{x}$ is a nondegenerate critical point with vanishing linear index and quadratic index equal to $k$, then $\bar{x}$ is a strongly stable Kuhn-Tucker point with s. index equal to $k$.

The proof of the next lemma can be given with the aid of Hager's theorem on Lipschitz continuity (cf. [6]) in a similar way as in [2, Theorem 2.2].

LEMMA 2.7. Let $\mathcal{O} \subset \mathbf{R}^{n}$ be open, $K$ a finite set and $f_{k}: \mathcal{O} \rightarrow \mathbf{R}^{p}$ locally Lipschitzian for every $k \in K$. Let $f: \mathcal{O} \rightarrow \mathbf{R}^{p}$ be a continuous selection of $\left\{f_{k}, k \in K\right\}$, i.e., $f$ is continuous and for every $x \in \mathcal{O}$ we have $f(x)=f_{k}(x)$ for some $k \in K$. Then $f$ is locally Lipschitzian.

Definition 2.2. Let $\mathcal{O}$ be an open subset of $\mathbf{R}^{n}$ and $\xi: \mathcal{O} \rightarrow \mathbf{R}^{n}$ a map. Then, $\boldsymbol{\xi}$ is called a vector field on $\mathcal{O}$. Consider the initial value problem:

$$
\frac{d x}{d t}=\xi(x) ; x(0)=\bar{x} .
$$

If a solution $x(t)$ of (2.3) uniquely exists up to the given integration time $\vec{t}$, then we denote $x(\bar{t})$ by $\Phi(\bar{x}, \bar{t})$. The map $\Phi$ is called the flow of the vectorfield $\xi$.

LemMa 2.8. Let $\mathcal{O} \subset \mathbf{R}^{n}$ be open and $\xi: \mathcal{O} \rightarrow \mathbf{R}^{n}$ a vectorfield. If $\xi$ is locally Lipschitzian, or $C^{k}(k \geqslant 1)$ respectively, then the corresponding flow $\Phi$ is defined on an open neighborhood of $\mathcal{O} \times\{0\}$, and $\Phi$ is continuous, or $C^{k}$ respectively. Moreover, if $\mathcal{O}=\mathbf{R}^{n}$, $\xi$ locally Lipschitzian and bounded, then $\Phi$ is defined on $\mathbf{R}^{n} \times \mathbf{R}$ (i.e., $\xi$ is “completely integrable" on $\mathbf{R}^{n}$ ).

For the local part of Lemma 2.8 (continuity etc. of $\Phi$ ) we refer to [9], whereas the globalization $\left(\mathcal{O}=\mathbf{R}^{n}\right.$ etc.) runs along the same lines as exposed in [11].

Lemma 2.9. Let $g_{j} \in C^{1}\left(\mathbf{R}^{n}, \mathbf{R}\right), j \in J$, and $\mathcal{O}$ an open subset of $\mathbf{R}^{n}$. Let $\bar{x} \in M[G] \cap \mathcal{O}$ and $\rho(\bar{x})=0$, where $\rho(x)=\min _{j \in J} g_{j}(x)$. Finally, suppose that $\xi: \mathcal{O} \rightarrow \mathbf{R}^{n}$ is a $C^{1}$-vector field with flow $\Phi(x, t)$, that $\xi(\bar{x})$ is an $M F$-vector at $\bar{x}$, and that the point $\hat{x} \in \mathcal{O}$ lies on the same integral curve of $\xi$ as the point $\bar{x}$, i.e., $\hat{x}=\Phi(\bar{x},-\bar{t})$ for some $\bar{i} \in \mathbf{R}$. Then there exist a neighborhood $\mathscr{V}$ of $\hat{x}$, a real number $\alpha>0$ and a unique Lipschitzian function $T: \mathscr{V} \rightarrow[\bar{t}-\alpha, \bar{t}+\alpha]$ satisfying:

(i) $\Phi(x, t)$ is well-defined on $\mathscr{V} \times[t-\alpha, t+\alpha]$, 
(ii) for $t \in[\bar{t}-\alpha, \bar{t}+\alpha]$ the composite function $\rho[\Phi(x, t)]$ vanishes exactly at $t=T(x)$ for all $x \in \mathscr{V}$ and changes sign,

(iii) in particular, all $g_{j}, j \in J_{0}(\bar{x})$, change sign along the integral curve through $\bar{x}$.

Proof. The fact, that $\xi(\bar{x})$ is an $M F$-vector at $\bar{x}$ implies immediately, that $g_{j}(\Phi(\bar{x}, t))>0$ (resp. $<0$ ) for all $j \in J_{0}(\bar{x})$ and all sufficiently small positive (resp. negative) $t$. We consider the case $\hat{x} \neq \bar{x}$ (the proof in case $\hat{x}=\bar{x}$ running along analogous lines) and $\bar{t}>0$. Then, for $\varepsilon>0$ sufficiently small, we have $\rho(\Phi(\hat{\mathrm{x}}, \bar{t}-\varepsilon))<0$ and $\rho(\Phi(\hat{x}, \bar{t}+\varepsilon))>0$. But then there exists a neighborhood $\mathscr{V}$ of $\hat{x}$ such that $\rho[\Phi(x, t)]$ traverses zero for all $x \in \mathscr{V}$ and $t$ sufficiently close to $\bar{t}$, the integration time $T$ needed for traversing the zero level of $\rho$ being unique, because $\xi$ is of course an $M F$-vector in a neighborhood of $\bar{x}$. Moreover, $T$ is easily seen to be continuous. Note that (reducing the neighborhood $\mathscr{V}$ if necessary) $T$ is a continuous selection from the functions $T_{j}, j \in J_{0}(\bar{x})$, each $T_{j}$ denoting the integration time (close to $\bar{t}$ ) needed for $g_{j}(\Phi(x, t))$ to vanish at $t=T_{j}(x)$. From the implicit function theorem it becomes obvious that $T_{J}$, $j \in J_{0}(\bar{x})$ is of class $C^{1}$ (cf. [11]). Since a $C^{1}$-function is locally Lipschitz continuous, Lemma 2.7 can be applied, which completes the proof.

The standard $k$-cell $D^{k}$ and its boundary $S^{k-1}$ are defined as follows:

$$
D^{k}=\left\{x \in \mathbf{R}^{k} \mid\|x\| \leqslant 1\right\}, S^{k-1}=\left\{x \in \mathbf{R}^{k} \mid\|x\|=1\right\} .
$$

For definitions etc. from algebraic topology we refer to [11] and [24].

LEMMA 2.10. Let $0<k<n$ and

$$
\sum_{n, k}:=\left\{x \in \mathbf{R}^{n} \mid-\sum_{i=1}^{k} x_{i}^{2}+\sum_{j=k+1}^{n} x_{j}^{2}=0\right\} .
$$

Then $\Sigma_{n, k} \cap \mathcal{O}$ is not a topological manifold, where $\mathcal{O}$ is any open neighborhood of the origin.

Proof. Put $p(x)=-\sum_{i=1}^{k} x_{i}^{2}+\sum_{j=k+1}^{n} x_{j}^{2}$. Note that $D p(x)=0$ if and only if $x=0$. Hence the set $\Sigma_{n, k} \backslash\{0\}$ is a differentiable manifold. So, the only point to be investigated is the origin and we may put $\mathcal{O}=\mathbf{R}^{n}$. We consider the case $n>2$, the lemma in case $n=2$ being obvious. If $x \in \Sigma_{n, k}$, then $\lambda x \in \Sigma_{n, k}$ for all $\lambda \in \mathbf{R}$. Consequently $\Sigma_{n, k}$ is contractible in view of the map $r(t, x)=(1-t) x$. In particular, $\Sigma_{n, k}$ has the homotopy type of a point. In parametric form ( $\varepsilon=$ parameter) a point $x$ belongs to $\Sigma_{n, k}$ if and only if the following two equations are simultaneously satisfied for some $\varepsilon \geqslant 0$ :

$$
\sum_{i=1}^{k} x_{i}^{2}=\varepsilon, \quad \sum_{j=k+1}^{n} x_{j}^{2}=\varepsilon .
$$


From (2.5) and the fact that $(0, \infty)$ is homeomorphic to $\mathbf{R}$ we see that $\Sigma_{n, k} \backslash\{0\}$ is homeomorphic to $S^{k-1} \times S^{n-k-1} \times \mathbf{R}$. Hence $\Sigma_{n, k} \backslash\{0\}$ has the homotopy type of the product manifold $S^{k-1} \times S^{n-k-1}$. Now suppose that $\sum_{n, k}$ is a topological manifold. Then its dimension equals $n-1$.

Case 1. $k=1$ or $k=n-1$. Then $\sum_{n, k} \backslash\{0\}$ consists of two connected components. Now $\Sigma_{n, k}$ is connected and $\operatorname{dim}\left(\Sigma_{n, k}\right) \geqslant 2$. But then deleting one point from $\sum_{n, k}$ cannot disconnect $\sum_{n, k}$, a contradiction.

Case 2. $1<k<n-1$. Now we derive a contradiction by means of generalizing the usual connectedness arguments. In fact, let $\pi_{r}$ denote the $r$ th homotopy group. Since $\Sigma_{n, k}$ is contractible, we have $\pi_{r}\left(\Sigma_{n, k}\right)=0$ for all $r \geqslant 1$. Moreover, since $\sum_{n, k}$ is assumed to be a manifold of dimension $n-1$, we have

$$
\pi_{r}\left(\sum_{n, k} \backslash\{0\}\right) \cong \pi_{r}\left(\sum_{n, k}\right) \text { for all } 1 \leqslant r<n-2
$$

So we obtain:

$$
\pi_{r}\left(\sum_{n, k} \backslash\{0\}\right)=0 \text { for all } 1 \leqslant r<n-2 .
$$

Next we show, in particular, that $\pi_{k-1}\left(\sum_{n, k} \backslash\{0\}\right) \neq 0$, which yields a contradiction with (2.6) since $1 \leqslant k-1<n-2$. To this aim we use the following two facts from homotopy theory (cf. [24]):

$$
\left.\begin{array}{l}
\pi_{r}\left(S^{r}\right) \cong \mathbf{Z}, r \geqslant 1 \\
\pi_{r}(X \times Y) \cong \pi_{r}(X) \times \pi_{r}(Y) \quad \text { (product rule) }
\end{array}\right\} .
$$

Since $\Sigma_{n, k} \backslash\{0\}$ has the homotopy type of $S^{k-1} \times S^{n-k-1}$ and $k-1 \geqslant 1$, we see with (2.7) that $\pi_{k-1}\left(\Sigma_{n, k} \backslash\{0\}\right) \neq 0$.

\section{Proof of Theorems A, B, C and comments}

\section{Proof of Theorem A.}

Without loss of generality we may omit the equality constraints (cf. Lemma 2.1 and Remark 2.1). The case $J_{0}(\bar{x})=\varnothing$ being trivial, let $\bar{x} \in M[G]$ with $J_{0}(\bar{x}) \neq \varnothing$ and choose an $M F$-vector $\xi \in \mathbf{R}^{n}$ at $\bar{x}$. In a sufficiently small, open neighborhood $\mathcal{O}$ of $\bar{x}$ we consider the vector $\xi$ as a constant vectorfield, and we may assume that $\xi$ is an $M F$-vector at all points of $\left\{x \in \mathbf{R}^{n} \mid \min _{j \in J} g_{j}(x)=0\right\} \cap \mathcal{O}$.

Since the integral curve of the constant vector-field $\xi$ through $\bar{x}$ intersects the zero set of each $g_{j}, j \in J_{0}(\bar{x})$ exactly at $\bar{x}$ (cf. Lemma 2.9), we can choose a point $\tilde{x}$ on it, near $\bar{x}$ with $g_{j}(\tilde{x})<0, j \in J_{0}(\bar{x})$. Without loss of generality we assume $\tilde{x}=0$ and $\xi=(1,0, \ldots, 0)^{T}$. For $x$ near the origin, the function $T$, as in Lemma 
2.9, is well-defined. Let $\Phi(x, t)$ denote the flow of the constant vectorfield $\xi$ and put $\psi\left(x_{1}, \ldots, x_{n}\right)=\Phi\left(\left(x_{1}, x_{2}, \ldots, x_{n}\right), T\left(0, x_{2}, \ldots, x_{n}\right)\right)$. Then, $\psi$ sends an open neighborhood $\mathscr{V}$ of the origin homeomorphically on an open neighborhood $\mathscr{U}$ of $\bar{x}$ and, moreover, the set $\left\{\left(x_{1}, \ldots, x_{n}\right) \mid x_{1} \geqslant 0\right\} \cap \mathscr{V}$ is mapped onto $M[G] \cap \mathscr{U}$.

Note. In the proof of Theorem B we sometimes use the expression "we add locally at $\bar{x}$ a function $g$ to the function $h$ ". By this expression we mean that we actually add the function $g(x) \cdot \zeta(x)$ to $h(x)$, where $\zeta(x)$ is a smooth function having the properties:

(i) $0 \leqslant \zeta(x) \leqslant 1$,

(ii) $\zeta$ has a compact support,

(iii) $\zeta(x)$ is identically equal to one in some neighborhood of $\bar{x}$.

\section{Proof of Theorem B.}

Sufficiency-part. Suppose that (MFCQ) holds at every point $x \in M[H, G]$. First we treat the case without equality constraints, i.e., $I=\varnothing$. Next we indicate that, with respect to $C_{S}^{1}$-perturbations, the general consideration can be reduced to the special case where the equality constraints remain unchanged. In the latter case, $C_{S}^{1}$-perturbations of the inequality constraints induce perturbations of the feasible set on a $C^{2}$-manifold (being a subset of the common zero set of the equality constraint functions) instead of $\mathbf{R}^{n}$.

Case 1: $I=\varnothing$. Let $\partial M[G]$ denote the boundary of $M[G]$ (cf. Theorem A). At $\bar{x} \in \partial M[G]$ we can choose $\xi_{\bar{x}}$ which is an $M F$-vector at $\bar{x}$. By continuity there exists an open neighborhood $\mathcal{O}_{\bar{x}}$ such that $\xi_{\bar{x}}$, regarded as a constant vectorfield on $\mathcal{O}_{\bar{x}}$, is an $M F$-vector at all points $x \in \partial M[G] \cap \mathcal{O}_{\bar{x}}$. Then we cover the set $\partial M[G]$ with these sets $\mathcal{O}_{\bar{x}}, \bar{x} \in \partial M[G]$, thus obtaining the open neighborhood $\mathcal{O}$ of $\partial M[G]$, where $\mathcal{O}=\bigcup_{\bar{x} \in \partial M[G]}^{\mathcal{O}_{\bar{x}}}$.

With the aid of a $C^{1}$-partition of unity subordinate to the covering $\left\{\mathcal{O}_{\bar{x}}, \bar{x} \in\right.$ $\partial M[G]\}$ of $\mathcal{O}$ (cf. [11]), by picking on each $\mathcal{O}_{\bar{x}}$ the constant vector $\xi_{\bar{x}}$ and subsequently glueing them together, we obtain a $C^{1}$-vectorfield $\xi$ on $\mathcal{O}$ with the property that $\xi(x)$ is an $M F$-vector at every point $x \in \partial M[G]$. The latter property follows from two facts: first, exploiting a partition of unity means that one takes (finite) convex combinations of the corresponding vectors $\xi_{\bar{x}}$ in some neighborhood of every point of $\mathcal{O}$; secondly, if $\xi^{k}$ is an $M F$-vector at $\bar{x} \in \partial M[G]$ for $k=1, \ldots, r$, then every convex combination of these vectors is an $M F$-vector at $\bar{x}$, too. By reducing the open neighborhood $\mathcal{O}$ of $\partial M[G]$ if necessary, we may assume that $\mathcal{O}$ is bounded (recall that $M[G]$ is assumed to be compact). Note that $\xi(x)$ does not vanish on $\mathcal{O}$. Then the normed vector field $\eta(x):=\xi(x) /\|\xi(x)\|$ is well-defined on $\mathcal{O}$ and $\eta$ is of class $C^{1}$. Let $\Phi(x, t)$ denote the flow of $\eta$. From 
Lemma 2.8 we learn that $\Phi$ is of class $C^{1}$, hence continuous. Consequently, noting that $\partial M[G]$ is compact, there exists an $\alpha>0$ such that $\Phi$ is defined on $\partial M[G] \times[-3 \alpha, 3 \alpha]$. For $\beta \in(0,3 \alpha]$ we define:

$$
\mathscr{N}_{\beta}=\{\Phi(x, t) \mid x \in \partial M[G], t \in(-\beta, \beta)\} .
$$

From Lemma 2.9 it follows that $\mathscr{N}_{\beta}$ is an open set and hence an open neighborhood of $\partial M[G]$. Using a continuity argument, we choose a $C_{S}^{1}$-neighborhood $\mathscr{V}$ of $G=\left(g_{1}, \ldots, g_{s}\right)$ such that the following two properties hold for any $\tilde{G}=\left(\tilde{g}_{1}, \ldots, \tilde{g}_{s}\right) \in \mathscr{V}($ cf. Lemma 2.2):

$$
\begin{aligned}
& M^{*}[\tilde{G}] \subset \mathscr{N}_{\alpha}, \text { where } \\
& M^{*}[G]=\left\{x \in \mathbf{R}^{n} \mid \min _{j \in J} \tilde{g}_{j}(x)=0\right\} ;
\end{aligned}
$$

Note that Theorem A and (P2) imply $\partial M[\tilde{G}]=M^{*}[\tilde{G}]$. Now, choose $\tilde{G} \in \mathscr{V}$. We show that $M[G]$ and $M[\tilde{G}]$ are homeomorphic.

Let $\varphi: \partial M[G] \rightarrow \partial M[\tilde{G}]$ be defined by sending $\bar{x} \in \partial M[G]$ to the unique intersection point of the trajectory through $\bar{x}$ of the vectorfield $\eta$ and the set $\partial M[\tilde{G}]$. Then $\varphi$ is easily seen to be bijective. To $\bar{x} \in \partial M[G]$ we assign the real number $T(\bar{x})$ satisfying $\Phi(\bar{x}, T(\bar{x}))=\varphi(\bar{x})$. Now, $T$ extends uniquely to a function on $\mathscr{N}_{2 \alpha}$ by defining $T(x)=T(\bar{x})$ if $x$ and $\bar{x}$ lie on the same trajectory of $\eta$. Lemma 2.9 implies $T$ to be locally Lipschitzian on $\mathscr{N}_{2 \alpha}$. In this way we obtain the following locally Lipschitzian vectorfield $\tilde{\eta}$ on $\mathscr{N}_{2 \alpha}$.

$$
\tilde{\eta}(x)=T(x) \cdot \eta(x), \quad x \in \mathscr{N}_{2 \alpha} .
$$

The two sets $\mathscr{N}_{2 \alpha}$ and $\mathbf{R}^{n} \backslash \overline{\mathcal{N}}_{\alpha}$ form an open covering of $\mathbf{R}^{n}$ ("- " denoting the closure). On the latter set we define the zero vectorfield and on $\mathscr{N}_{2 \alpha}$ we consider the vectorfield $\tilde{\eta}$. By means of a $C^{1}$-partition of unity subordinate to the former covering we glue the two vectorfields together and obtain a bounded, locally Lipschitzian vectorfield $\zeta$ on $\mathbf{R}^{n}$ which coincides with $\tilde{\eta}$ on $\mathscr{N}_{\alpha}$. Let $\psi(x, t)$ denote the flow of $\zeta$. Then $\psi(x, t)$ is continuous on $\mathbf{R}^{n} \times \mathbf{R}$ (cf. Lemma 2.8) and for each $t$ we have of course: $\psi(\cdot, t): \mathbf{R}^{n} \rightarrow \mathbf{R}^{n}$ is a homeomorphism of $\mathbf{R}^{n}$ onto itself. In view of the very construction of the vectorfield $\zeta$ we finally obtain:

$$
\psi(M[G], 1)=M[\tilde{G}],
$$

and the proof in the case of $I=\varnothing$ is complete.

Case 2: $I \neq \varnothing$. First we consider the special case without inequality constraints, i.e., $J=\varnothing$. The feasible set $M[H]$ is a compact $C^{2}$-manifold (cf. Remark 2.1) then. Given a neighborhood $\mathcal{O}$ of $M[H]$ we can choose a $C_{S}^{1}$-neighborhood $\mathscr{V}$ of $H=\left(h_{1}, \ldots, h_{m}\right)$ in $C^{2}\left(\mathbf{R}^{n}, \mathbf{R}\right)^{m}$ such that for each $\tilde{H} \in \mathscr{V}$ the 
following properties hold with respect to the linear homotopy

$$
\mathscr{H}(x, t):=(1-t) H(x)+t \tilde{H}(x):
$$

(A1) The set $\mathscr{M}:=\left\{(x, t) \in \mathbf{R}^{n} \times[0,1] \mid \mathscr{H}(x, t)=0\right\}$ is compact and $\mathscr{M} \subset \mathcal{O}$ $\times[0,1]$;

(A2) At every point $(\bar{x}, \bar{t}) \in \mathscr{M}$ the Jacobian $D_{x} \mathscr{H}(\bar{x}, \bar{t})$, as a linear map from $\mathbf{R}^{n}$ to $\mathbf{R}^{m}$, is surjective.

Note that $\mathscr{M}$ is a $C^{2}$-manifold with boundary and we can regard the parameter $t$ as a function on $\mathscr{M}$. Then, the level sets of $\left.t\right|_{\Perp}$ are "intermediate feasible sets", in particular, the 0 -level, or 1-level of $\left.t\right|_{\mathscr{M}}$, respectively, equals $M[H]$, or $M[\tilde{H}]$, respectively. Moreover, for $t \in(0,1)$ there are no critical points for $\left.t\right|_{\mathscr{M}}$. Recall that $\mathscr{M}$ is of class $C^{2}$; hence, the tangent spaces depend $C^{1}$ on the points of $\mathscr{M}$. But then, with the aid of a $C^{1}$-vectorfield tangent to $\mathscr{M}$ and induced by the orthogonal projection of $(0, \ldots, 0,1)^{T}(=$ gradient of $t$ as a function of $(n+1)$ variables) on the tangent spaces of $\mathscr{M}$, we can integrate the 0 -level of $\left.t\right|_{\mathscr{M}}$ with integration time equal to one up to the 1 -level. In this way we obtain a $C^{1}$-diffeomorphism of $M[H]$ onto $M[\tilde{H}]$ (see [10] and, in particular, [14], [16, Chapter 6] for details on the above construction).

From the above construction we may conclude that in the general case $(I \neq \varnothing, J \neq \varnothing)$ we may restrict ourselves to $C_{S}^{1}$-perturbations of the inequality constraint functions only, thus leaving the equality constraints unchanged. But then, in a neighborhood of the feasible set $M[H, G]$, the common zero set of the equality constraint functions is a $C^{2}$-manifold (cf. Remark 2.1) and we can copy the argument from Case 1 in local coordinates; see, for example, [11] for details on such constructions. This completes the sketch of the proof of the sufficiency part.

Necessity-Part. Suppose that (MFCQ) fails to hold at $\bar{x} \in M[H, G]$. By means of an initial $C_{S}^{1}$-approximation (cf. Lemma 2.3) we may assume that the functions $h_{\imath}, g$, are of class $C^{\infty}$ and, moreover, that the functional values as well as the first partial derivatives at the point $\bar{x}$ coincide with the original ones. Now the main idea is to approximate $H, G$ (arbitrarily well in the $C_{S}^{1}$-sense) by means of functions $\tilde{H}, \tilde{G}$, resp. $\hat{H}, \hat{G}$, such that $M[\tilde{H}, \tilde{G}]$ is not homeomorphic with $M[\hat{H}, \hat{G}]$. For this purpose we distinguish whether the Jacobian $D H(\bar{x})$, considered as a linear map from $\mathbf{R}^{n}$ to $\mathbf{R}^{m}$, is surjective or not. Without loss of generality we assume that $\bar{x}=0$ in the sequel.

Case 1: $D H(\bar{x})$ is not surjective. If $J_{0}(\bar{x}) \neq \varnothing$, we add locally at $\bar{x}$ a small positive constant to the functions $g_{j}, j \in J_{0}(\bar{x})$, and we obtain that $\bar{x}$ remains feasible after this perturbation but $J_{0}(\bar{x})=\varnothing$. Next, we add locally at $\bar{x}(=0)$ linear functions (with arbitrary small derivatives) to the functions $h_{i}, i \in I$, such that, after this perturbation, we have at $\bar{x}$ :

$$
\operatorname{rank}\left(D^{T} h_{1}|\cdots| D^{T} h_{m}\right)=\operatorname{rank}\left(D^{T} h_{1}|\cdots| D^{T} h_{m-1}\right)=m-1 .
$$


Then, in a neighborhood of $\bar{x}$ the common zero set of the functions $h_{1}, \ldots, h_{m-1}$, denoted by $M[m-1]$ is a smooth manifold and moreover, the point $\bar{x}$ is a critical point for $h_{m \mid M[m-1]}$. Then, we add locally at $\bar{x}(=0)$ a homogeneous polynomial of degree two (with arbitrary small coefficients) to the function $h_{m}$ in order that, after this perturbation, the point $\bar{x}$ is a nondegenerate critical point for $h_{m \mid M[m-1]}$ (cf. Definition 2.1). Finally outside a sufficiently small neighborhood of $\bar{x}$ we approximate the functions $h_{l}, g_{j}$ in the $C_{S}^{1}$-sense by means of $\tilde{h}_{t}, \tilde{g}_{j}$, such that the set $M[\tilde{H}, \tilde{G}]$ is compact and, except at $\bar{x}$, the linear independence condition holds on $M[\tilde{H}, \tilde{G}]$ (cf. Lemma 2.4). Note that the feasible set $M[\tilde{H}, \tilde{G}]$ coincides with $M[H]$ in a neighborhood of $\bar{x}$. We distinguish two subcases.

Subcase 1.a. The point $\bar{x}$ is a local minimum (resp. local maximum) for $\tilde{h}_{m \mid M[m-1]}$ (recall that $h_{m}$ and $\tilde{h}_{m}$ coincide in a neighborhood of $\bar{x}$ ). Since $\bar{x}$, as a nondegenerate critical point, is isolated, we see that $\bar{x}$ is an isolated feasible point. If we add locally at $\bar{x}$ a small negative (resp. positive) constant to the function $\tilde{h}_{m}$, thereby obtaining the function $\hat{h}_{m}$, we get: $M[\tilde{H}, \tilde{G}]=M[\hat{H}, \hat{G}] \cup$ $\{\bar{x}\}$ (disjoint union), where $\hat{h}_{t}=\tilde{h}_{t} i \neq m$, and $\hat{G}=\tilde{G}$. But then the number of connected components of $M[\hat{H}, \hat{G}]$ ( finite, since $M[\hat{H}, \hat{G}]$ is a compact topological manifold with boundary) is one less than the corresponding number for $M[\tilde{H}, \tilde{G}]$. Consequently, $M[\tilde{H}, \tilde{G}]$ and $M[\hat{H}, \hat{G}]$ cannot be homeomorphic.

Subcase 1.b. The point $\bar{x}$ is neither a local minimum, nor a local maximum for $\tilde{h}_{m \mid M[m-1]}$. Then, application of the Morse Lemma, in local coordinates for $M[m-1]$ (cf. [11]), yields that the feasible set $M[\tilde{H}, \tilde{G}]$ in a neighborhood of $\bar{x}$ is represented by means of the following set $\sum$ in $\mathbf{R}^{n-m+1}$ :

$$
\sum=\left\{u \in \mathbf{R}^{n-m+1} \mid \sum_{i=1}^{n-m+1} \pm u_{\imath}^{2}=0\right\},
$$

where at least one positive as well as one negative square appears in (3.1). But then Lemma 2.10 tells us that $\Sigma$ is not the germ of a topological manifold at $u=0$. Next we add an arbitrarily small positive constant to $\tilde{h}_{m}$ locally at $\bar{x}$ and we obtain, in view of (3.1), that the linear independence condition is satisfied at all points of $M[\hat{H}, \hat{G}]$ and hence $M[\hat{H}, \hat{G}]$ is a topological manifold with boundary. Here $\hat{h}_{i}=\tilde{h}_{i}, i \neq m, \hat{h}_{m}$ is the perturbed $\tilde{h}_{m}$ and $\hat{G}=\tilde{G}$.

Consequently we conclude that $M[\hat{H}, \hat{G}]$ is not homeomorphic with $M[\tilde{H}, \tilde{G}]$.

Case 2: $D H(\bar{x})$ is surjective. In this case the common zero set of the functions $h_{1}, \ldots, h_{m}$ is obviously a smooth manifold in a neighborhood of $\bar{x}$ and so for a further local analysis we may assume $I=\varnothing$ (cf. Remark 2.1).

Since the (MFCQ) is not satisfied at $\bar{x}$, it follows that the linear system

$$
D g_{j}(\bar{x}) \cdot \xi>0, \quad j \in J_{0}(\bar{x})
$$


is not solvable. But then, in virtue of Farkas' Lemma, the following dual system has a solution:

$$
\left.\sum_{j \in J_{0}(\bar{x})} u_{j} D g_{j}(\bar{x})=0, \quad u_{j} \geqslant 0, \quad \begin{array}{r}
j \in J_{0}(\bar{x}), \\
\sum_{j \in J_{0}(\bar{x})} u_{j}=1
\end{array}\right\} .
$$

Next we choose a minimal subset $\left\{D_{g_{j}}(\bar{x}), j \in \tilde{J}\right\}$, where $\tilde{J} \subset J_{0}(\bar{x})$, such that (3.3) is satisfied, replacing $J_{0}(\bar{x})$ by $\tilde{J}$. The corresponding numbers $u$, will be denoted by $\bar{u}_{j}, j \in \tilde{J}$. Note that the numbers $\bar{u}_{j}, j \in \tilde{J}$, are strictly positive. Now we add locally at $\bar{x}$ a small positive constant to the functions $g_{j}, j \in J_{0}(\bar{x}) \backslash \tilde{J}$. After this perturbation the feasible set is, locally around $\bar{x}$, equal to the following set:

$$
\left\{x \mid \max _{j \in \tilde{J}} f_{j}(x) \leqslant 0\right\}
$$

where $f_{j}=-g_{j}$.

We proceed by perturbing exactly one of the functions $f_{j}, j \in \tilde{J}$, locally at $\bar{x}(=0)$ by means of a homogeneous polynomial of degree two (arbitrarily small coefficients) such that the matrix $D:=V^{T}\left(\Sigma_{j \in j} \bar{u}_{j} D^{2} f_{j}(\bar{x})\right) V$ is nonsingular after the perturbation, where $V$ is a matrix whose columns are $n$-vectors forming a basis for the linear space $\left\{\xi \in \mathbf{R}^{n} \mid D f_{j}(\bar{x}) \xi=0, j \in \tilde{J}\right\}$ of dimension $n-|\tilde{J}|+1$. Let $k$ denote the number of negative eigenvalues of the above matrix $D$. Outside a small neighborhood of $\bar{x}$ we may assume (cf. Lemma 2.4) that (LI) holds at the feasible points.

Now we are in the following situation: in a neighborhood of $\bar{x}$ the feasible set has the structure of a lower level set of a function of maximum type in a neighborhood of a nondegenerate (+)-Kuhn-Tucker point of quadratic index $k$ (cf. [11], Chapter 4).

But then, in the spirit of Theorem 4.2.2 in [11], we may conclude:

The local addition of an (arbitrarily) small positive resp. negative constant to all functions $f_{j}, j \in \tilde{J}$, gives rise to two feasible sets $M[\tilde{H}, \tilde{G}]$ and $M[\hat{H}, \hat{G}]$, respectively, both of them compact and satisfying (LI) at all the feasible points, with the property that $M[\tilde{H}, \tilde{G}]$ is homotopy-equivalent to $M[\hat{H}, \hat{G}]$ with a $k$-cell attached (here we take, without loss of generality, the equality constraints into account again). But then (cf. [11]), $M[\tilde{H}, \tilde{G}]$ and $M[\hat{H}, \hat{G}]$ do not have the same homotopy type as compact topological manifolds with boundary. As a consequence, they cannot be homeomorphic.

This completes the proof of Theorem B.

REMARK 3.1. In the formulation of the necessity-part of Theorem $B$ it suffices that $h_{i}$ is of class $C^{1}$ instead of $C^{2}, i \in I$. 

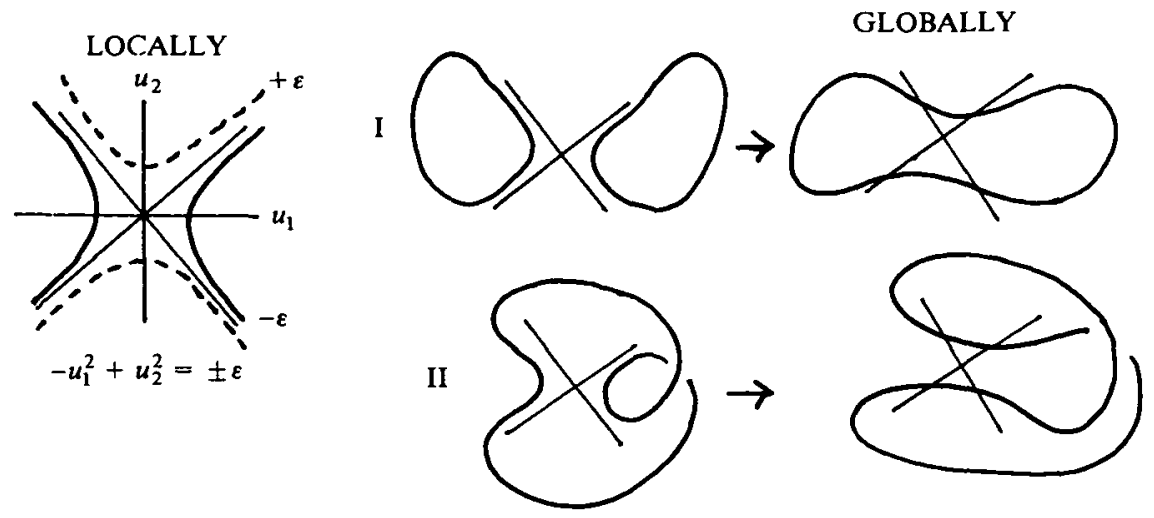

Figure 3.1

Remark 3.2. In the necessity-part, Subcase 1.b, we cannot simply compare "regular" levels $\pm \varepsilon(\varepsilon>0)$ for the function $\Sigma \pm u_{i}^{2}$ (cf. (3.1)). In fact, globally the resulting sets $M[\tilde{H}, \tilde{G}]$ and $M[\hat{H}, \hat{G}]$ might even be diffeomorphic in case the number of negative and positive squares in (3.1) coincide. Compare Figure 3.1 for a picture of this phenomenon in case $n=3, m=2$ and $J=\varnothing$ (situation II).

Remark 3.3. Note that the sets $M[H, G]$ and $M[\tilde{H}, \tilde{G}]$ in the sufficiency part of Theorem B are even isotopic, in the sense that $M[H, G]$ is transformed continuously into $M[\tilde{H}, \tilde{G}]$ by means of a continuous one-parameter family of homeomorphisms on $\mathbf{R}^{n}$. Isotopy is stronger than homeomorphy; in fact, the sets $S_{1}, S_{2} \subset \mathbf{R}^{2}$ are homeomorphic but not isotopic in $\mathbf{R}^{2}$, where

$$
\begin{aligned}
& S_{1}=\{(0,0)\} \cup\left\{x \in \mathbf{R}^{2} \mid\|x\|=1\right\}, \\
& S_{2}=\{(2,0)\} \cup\left\{x \in \mathbf{R}^{2} \mid\|x\|=1\right\} .
\end{aligned}
$$

REMARK 3.4. The sufficiency part of Theorem B remains (obviously by construction) true if we replace the embedding space $\mathbf{R}^{n}$ by some compact neighborhood $K$ of $M[H, G]$ and if we subsequently restrict our considerations to this set $K$. Then the $C_{S}^{1}$-perturbations can be replaced just by perturbations of the functions $h_{i}, g_{j}$, up to derivatives of first order on the compact set $K$ by means of a positive constant $\varepsilon$.

REMARK 3.5. In Theorem B we assumed $M[H, G]$ to be compact. For unbounded $M[H, G]$ the necessity part becomes delicate. To see this, consider a countably infinite discrete subset $A$ of $\mathbf{R}$. Take a point $a \in A$. Then $A$ and 
$A \backslash\{a\}$ are homeomorphic just in view of the fact that the cardinalities of $A$ and $A \backslash\{a\}$ coincide. In the sufficiency part, one might consider unbounded $M[H, G]$ in so far as the vector fields constructed analogously are completely integrable.

\section{Proof of Theorem C.}

Part $a$. Let $\bar{x} \in M_{a}^{b}$ and consider the following auxiliary optimization problem $\mathscr{P}(\bar{x})$ :

$$
\mathscr{P}(\bar{x})\left\{\begin{array}{l}
\text { Minimize } \frac{1}{2}\|\xi\|^{2} \\
\text { Subject to: } \\
\begin{cases}D f(\bar{x}) \xi=-1, & \\
D h_{i}(\bar{x}) \xi=0, & i \in I, \\
D g_{j}(\bar{x}) \xi \geqslant 0, & j \in J_{0}(\bar{x}) .\end{cases}
\end{array}\right.
$$

Since $\bar{x}$ is not a Kuhn-Tucker point for $\left.f\right|_{M[H, G]}$, we see that the feasible set for $\mathscr{P}(\bar{x})$ is not empty. Further, from strict convexity of $\frac{1}{2}\|\xi\|^{2}$ as well as the convexity of the feasible set for $\mathscr{P}(\bar{x})$, we see that $\mathscr{P}(\bar{x})$ has exactly one solution which we will denote by $\xi(\bar{x})$. Of course $\xi(\bar{x})$ need not depend continuously on $\bar{x}$.

Let us proceed with the proof, assuming that the following contention has been proved:

Contention: $\xi(x)$ is bounded on $M_{a}^{b}$, i.e.,

$$
\sup _{x \in M_{a}^{b}}\|\xi(x)\| \leqslant K<\infty .
$$

Let $\bar{x} \in M_{a}^{b}$ and choose a local $C^{2}$-coordinate system given by $\Phi(x)$ as in Formula (2.1). Next consider $\tilde{\xi}(x)$ generated by $\hat{\xi}(\bar{x})$ and defined in an open neighborhood $\mathcal{O}_{\bar{x}}$ of $\bar{x}$ :

$$
\tilde{\xi}(x)=D \Phi^{-1}(\Phi(x)) \cdot D \Phi(\bar{x}) \cdot \hat{\xi}(\bar{x}),
$$

where $\hat{\xi}(\bar{x})=\xi(\bar{x})$ in case $J_{0}(\bar{x})=\varnothing$ and $\hat{\xi}(\bar{x})=\xi(\bar{x})+\xi^{*}, \xi^{*}$ being an $M F$-vector at $\bar{x}$ with $\left|D f(\bar{x}) \xi^{*}\right|<\frac{1}{4}$ otherwise.

Note that $\tilde{\xi}(x)$ is of class $C^{1}$ and that $\tilde{\xi}(x)$ is an $M F$-vector in a neighborhood (say, also $\mathcal{O}_{\bar{x}}$ ) of $\bar{x}$. In particular, $\tilde{\xi}(x)$ is tangent to the common zero set of the functions $h_{i}$, locally considered as a $C^{2}$-manifold. Moreover, we may assume that $\|\tilde{\xi}(x)\| \leqslant 2 K$ on $\mathcal{O}_{\bar{x}}$ (cf. the Contention). In this way we obtain an open covering of $M_{a}^{b}$ with vectorfields of the type $\tilde{\xi}$ for each covering element. Together with the zero-vectorfield on the open set $\mathbf{R}^{n} \backslash M_{a}^{b}$ and a $C^{1}$-partition of unity of $\mathbf{R}^{n}$, 
taking $\mathbf{R}^{n} \backslash M_{a}^{b}$ as a covering element into account, we obtain a vectorfield $\eta$ on $\mathbf{R}^{n}$ having the following properties:

(1) $\eta$ is of class $C^{1}$ and bounded (on $\mathbf{R}^{n}$ ) (hence, $\eta$ is completely integrable; cf.

Lemma 2.8),

(2) $\eta(x)$ is an $M F$-vector at all points of $M_{a}^{b}$,

(3) $D f(x) \eta(x) \leqslant-\frac{1}{2}$ for all $x \in M_{a}^{b}$,

(4) $\psi(\cdot, t)[M] \subset M$ for all $t \geqslant 0$, where $\psi$ denotes the flow of $\eta$.

Now, let $\bar{x} \in M_{a}^{b}$. Denote by $T(\bar{x})$ that value of $t$ for which $f[\psi(\bar{x}, T(\bar{x}))]=a$. Note that $T(\bar{x}) \leqslant 2(b-a)$ and it is easily verified that $T$ is continuous on $M_{a}^{b}$.

Finally, we define the mapping $r:[0,1] \times M^{b} \rightarrow M^{b}$ as follows:

$$
r(t, x)=\left\{\begin{array}{l}
x, \text { for } x \in M^{a}, \\
\psi[x, t \cdot T(x)], \quad \text { for } x \in M_{a}^{b} .
\end{array}\right.
$$

Then $r$ is continuous and $r(0, \cdot)$ is the identity. Moreover, as $t$ varies from zero to one, $M^{b}$ is continuously deformed (within $M^{b}$ ) to $M^{a}$, whereas all points of $M^{a}$ remain fixed. Hence $M^{a}$ is a strong deformation retract of $M^{b}$.

Finally, we have to prove the Contention. In fact, we will show that for all $\bar{x} \in M_{a}^{b}$ the following inequality holds:

$$
\|\xi(x)\|^{-1} \geqslant \inf \left\{d\left(D f(x), N_{x}\right) \mid x \in M_{a}^{b}\right\} .
$$

The right-hand-side of (3.6) is positive in view of the absence of Kuhn-Tucker points in $M_{a}^{b}$ and the validity of Condition $C^{*}$. So it suffices to show (3.6).

Let $\bar{x} \in M_{a}^{b}$. Since the constraints in (3.4) are linear, we have, in particular, that the unique point $\xi(\bar{x})$ is a Kuhn-Tucker point for $\mathscr{P}(\bar{x})$, i.e.:

$$
\xi(\bar{x})=\alpha D f(\bar{x})+\sum_{i \in I} \lambda_{i} D h_{i}(\bar{x})+\sum_{j \in J} \mu_{j} D g_{j}(\bar{x})
$$

where

$$
\tilde{J}=\left\{j \in J_{0}(\bar{x}) \mid D g_{j}(\bar{x}) \xi(\bar{x})=0\right\} \quad \text { and } \quad \mu_{j} \geqslant 0, j \in \tilde{J} .
$$

From (3.4) and (3.7) we see that $\|\xi(\bar{x})\|^{2}=-\alpha$. Since $\xi(\bar{x}) \neq 0$ (cf. (3.4)), we have $\alpha<0$. Then, noting that $|\alpha|=\|\xi(\bar{x})\|^{2},(3.7)$ implies:

$$
\|\xi(\bar{x})\|=\|\xi(x)\|^{2}\left\|D f(\bar{x})-\sum_{i \in I} \frac{\lambda_{i}}{-\alpha} D h_{i}(\bar{x})-\sum_{j \in J} \frac{\mu_{j}}{-\alpha} D g_{j}(\bar{x})\right\| .
$$

But now (3.6) follows from (3.8), since $\tilde{J} \subset J_{0}(\bar{x}),-\alpha>0$ and all $\mu_{j}$ are nonnegative.

Part $b$. In view of Part a, the actual deformation part, we can reduce the proof now to those techniques as explained in detail in [11] by means of a preliminary local perturbation at $\bar{x}$. In fact, take a closed ball $B$ with center $\bar{x}$ and small radius, and perturb $f, h_{i}, g_{j}$ in the $C^{2}$-sense in a neighborhood of $B$ such that (ii) and (iii) of Lemma 2.5 hold with $C_{2}=B$. 
Since $\bar{x}$ is strongly stable, the preceding perturbation replaces $\bar{x}$ by one new strongly stable point $\tilde{x}$ in $B$. But now, $\tilde{x}$ is a nondegenerate critical point with vanishing linear index and quadratic index equal to the s-index of $\bar{x}$ as a strongly stable Kuhn-Tucker point. So, together with the global deformation part (Part a), the cell-attaching takes place in local coordinates around $\tilde{x}$, which in fact reduces the present situation to well-known techniques (as in [11]).

Remark 3.6. The converse of Theorem C.a is not true, since $M^{a}$ might be a strong deformation retract from $\boldsymbol{M}^{b}$, even if $M_{a}^{b}$ contains Kuhn-Tucker points (in fact, create a pair of Kuhn-Tucker points with difference in $s$.index equal to one).

\section{Some consequences}

Theorem $\mathrm{C}$ implies, in particular, that the so-called Morse relations from critical point theory (relating certain combinations of nondegenerate critical points with the topology of the underlying manifold) remain valid for constrained minimization (under the relaxed assumptions of Theorem C). See [11] for a detailed exposé on the subject of Morse relations.

As an example, we state the following corollary:

Corollary 4.1. Let $f, h_{i}, g_{j} \in C^{2}\left(\mathbf{R}^{n}, \mathbf{R}\right), i \in I, j \in J$. Suppose that $M[H, G]$ is compact and that (MFCQ) is satisfied at all points $x \in M:=M[H, G]$; put $p=\operatorname{dim} M$ (cf. Theorem A). Suppose moreover, that all Kuhn-Tucker points of $f \mid M$ are strongly stable.

(i) If, in addition, $M$ is connected and $f \mid M$ has $\ell$ local minima, then there exist at least $\ell-1 K u h n-T u c k e r$ points for $f \mid M$ with s.index equal to one.

(ii) Let $c_{r}, r=0,1, \ldots, p$, denote the number of Kuhn-Tucker points for $f \mid M$ with s.index equal to $r$ and put $\gamma=\sum_{i=0}^{p}(-1)^{r} c_{r}$. Then $\gamma$ depends only on the homeomorphy-type of $M$. Moreover, $\gamma$ is constant on some $C_{S}^{2}$-neighborhood of $(f, H, G)$.

(iii) If $M$ is homeomorphic to the unit ball $D^{p}$, then the number of Kuhn-Tucker points for $f \mid M$ is odd.

(iv) If the number of Kuhn-Tucker points for $f \mid M$ is equal to one, then $M$ is homeomorphic to the unit ball $D^{p}$.

Proof. We can use the cell-attaching results from [11, Chapter 5], in view of Theorem C, thereby replacing every entry of a "nondegenerate (+)-Kuhn-Tucker point with quadratic index $r$ " by a "strongly stable Kuhn-Tucker point with $s$.index $r$ ". Now Theorem 5.2.2 and Remark 5.2.5 from [11] imply (i). Remark 
5.2.3 from [11] implies the first statement of (ii) since $\gamma$ turns out to be the Euler-characteristic of $M$, hence a homotopy- (and thus, homeomorphy-) invariant. Theorem $B$ then implies the second statement of (ii). Since $D^{p}$ is contractible, we have $\gamma\left(D^{p}\right)=1$, which implies (iii). It remains to prove (iv). First we may assume, after a $C_{S}^{2}$-perturbation, that (LI) is additionally satisfied at all points of $M$ and all critical points of $f \mid M$ are nondegenerate (Lemma 2.5 and Theorem B). Now the unique Kuhn-Tucker point, say $\bar{x}$, must be the global minimum for $f \mid M$. In view of the generalized Morse Lemma ([11], Theorem 3.2.1), for $\varepsilon>0$ sufficiently small the lower level set $M^{f(\bar{x})+\varepsilon}$ is homeomorphic to the set

$$
\left\{x \in \mathbf{R}^{p} \mid \sum_{i=1}^{q} x_{i}+\sum_{j=q+1}^{p} x_{j}^{2} \leqslant \varepsilon, x_{i} \geqslant 0, i=1, \ldots, q\right\} .
$$

But the latter set is easily seen to be homeomorphic to $D^{p}$. Let $c$ denote the maximal value of $f \mid M$. For $t \in[\varepsilon, c]$ we take $g_{t}(x):=t-f(x)$ as an additional inequality constraint and we see that (MFCQ) remains valid on $M(t):=M \cap$ $\{x \mid t-f(x) \geqslant 0\}$ for all $t \in[\varepsilon, c]$. But then, Theorem B and Remark 3.4 imply that the homeomorphy type of $M(t)$ is locally constant on $[\varepsilon, c]$, hence constant.

\section{Acknowledgement}

We would like to thank Bruce Craven, Stephen Robinson and the referee for their comments, which improved the first version of the manuscript. The paper originated in the spring of 1985 while $\mathrm{H}$. Th. Jongen held the chair "Karl Weierstrass" at the Humboldt University in Berlin.

\section{References}

[1] B. Bank, J. Guddat, D. Klatte, B. Kummer and K. Tammer, Non-linear parametric optimization (Akademie-Verlag, Berlin, 1982).

[2] A. L. Dontchev and H. Th. Jongen, "On the regularity of the Kuhn-Tucker curve", SIAM J. Control Optim. (to appear), (Preprint No. 460, Twente University of Technology (1984)).

[3] A. V. Fiacco and J. Kyparisis, "Sensitivity analysis in nonlinear programming under second order assumptions", in Systems and Optimization, Lect. Notes in Control and Inf. sciences, Vol. 66 (eds. A. Bagchi and H. Th. Jongen) (1985), 74-97.

[4] A. V. Fiacco and G. P. McCormick, Nonlinear programming: Sequential unconstrained mintmi zation techniques (Wiley, New York, 1968).

[5] J. Gauvin, "A necessary and sufficient regularity condition to have bounded multipliers in nonconvex programming", Math. Programming 12 (1977), 136-138. 
[6] W. W. Hager, "Lipschitz continuity for constrained processes", SIAM J. Control Optim. 17 (1979), 321-338.

[7] R. Hettich and H. Th. Jongen, "On first and second order conditions for local optima for optimization problems in finite dimensions", Methods of Operations Research Vol. 23 (1977), 82-97.

[8] M. W. Hirsch, Differential topology (Springer-Verlag, 1976).

[9] W. Hurewicz, Lectures on ordinary differential equations (The M. I. T. Press, 1958).

[10] H. Th. Jongen, P. Jonker and F. Twilt, "On one-parameter families of sets defined by (in)equality constraints", Nieuw Arch. Wisk. (3), XXX (1982), 307-322.

[11] H. Th. Jongen, P. Jonker and F. Twilt, Non-linear optimization in $\mathbf{R}^{n}$, I. Morse theory, Chebyshev approximation (Peter Lang Verlag, Frankfurt a. M., Bern, New York, 1983).

[12] H. Th. Jongen, P. Jonker and F. Twilt, "One-parameter families of optimization problems: Equality constraints", J. Optım. Theory Appl. 48 (1986), 141-161.

[13] H. Th. Jongen, P. Jonker and F. Twilt, "Critical sets in parametric optimization", Math. Programming 35 (1986), 1-21.

[14] H. Th. Jongen, P. Jonker and F. Twilt, "Nonlinear optimization theory in $\mathbf{R}^{n}$ from a global point of view V", Twente University of Technology, Preprint No. 462 (1984).

[15] H. Th. Jongen, P. Jonker and F. Twilt, "Nonlinear optimization theory in $\mathbf{R}^{n}$ from a global point of view VI", Twente University of Technology, Preprint No. 506 (1985).

[16] H. Th. Jongen, P. Jonker and F. Twilt, Nonlinear optimization in $\mathbf{R}^{n} ;$ II. Transversality, flows, parametric aspects (forthcoming, to appear in Peter Lang Verlag).

[17] M. Kojima, "Strongly stable stationary solutions in nonlinear programs", in Analysis and computation of fixed points (ed. S. M. Robinson), (Academic Press, New York, 1980), 93-138.

[18] M. Kojima and R. Hirabayashi, "Continuous deformation of nonlinear programs", Math. Programming Stud. 21 (1984), 150-198.

[19] E. S. Levitin (Е. C. Левитив), “О коррехция решений задач нелинейного программирования с непольной ивформапии", Всесопзная петняя пхола о методах огтимпзации и их применения (Akad. Nauk SSSR, Sib. Otdel, Irkutsk, 1974 (I, II)).

[20] J. Milnor, Morse theory, Annals of Mathematics Studies No. 51 (Princeton University Press, 1963).

[21] R. S. Palais and S. Smale, "A generalized Morse theory", Bull. Amer. Math. Soc. 70 (1964), 165-172.

[22] S. M. Robinson, "Stability theory for systems of inequalities, part II: differentiable nonlinear systems", SIAM J. Numer. Anal. Vol. 13, No. 4 (1976), 497-513.

[23] S. Schecter, "Structure of the first order solution set for a class of nonlinear programs with parameters", Math. Programming 34 (1986), 84-110.

[24] E. H. Spanier, Algebraic topology (McGraw-Hill, Inc., 1966). 